CLINICAL STUDY

\title{
Association of intramyocellular, intraperitoneal and liver fat with glucose tolerance in severely obese adolescents
}

\author{
Tero Saukkonen, Sami Heikkinen ${ }^{1}$, Antti Hakkarainen ${ }^{2}$, Anna-Maija Häkkinen ${ }^{3}$, Koen van Leemput ${ }^{2}$, \\ Marita Lipsanen-Nyman and Nina Lundbom ${ }^{3}$ \\ Hospital for Children and Adolescents, University of Helsinki, O0029 Helsinki, Finland, ${ }^{1}$ Laboratory of Organic Chemistry, University of Helsinki, \\ 00014 Helsinki, Finland, ${ }^{2}$ Medical Imaging Center and ${ }^{3}$ Department of Oncology, University of Helsinki, O0029 Helsinki, Finland
}

(Correspondence should be addressed to T Saukkonen who is now at Novo Nordisk Farma Oy, Hevosenkenkä 3, O2600 Espoo, Finland; Email: tskk@novonordisk.com)

\begin{abstract}
Objective: Impaired glucose tolerance (IGT) is common among obese adolescents. The aim of the present study was to investigate the association between glucose tolerance and intramyocellular, intra-abdominal and liver fat in adolescents presenting with early-onset severe obesity.

Design and methods: We studied 21 adolescents (mean age 13.5 years, range 11.5-15.9 years) referred to secondary care due to severe obesity (relative weight for height $>+60 \%$ or body mass index $>98$ th percentile for age and sex, before the age of 10 years) and their eight non-obese siblings (mean age 14.4 years, range 11.8-16.7 years). All subjects underwent oral glucose tolerance tests, followed by magnetic resonance spectroscopy (MRS) to measure the intramyocellular fat content in mainly oxidative soleus and mainly glycolytic tibialis anterior muscles. MRS was also used to measure liver fat. Abdominal fat (subcutaneous, intraperitoneal and retroperitoneal) was measured using MR imaging. Results: Compared with their non-obese siblings, the obese adolescents had increased fat deposition in all anatomic locations studied. Eight obese adolescents had IGT, and they also had increased intramyocellular fat in the soleus $(P=0.03)$ and increased intraperitoneal fat $(P=0.04)$ compared with obese subjects with normal glucose tolerance (NGT). In contrast, no significant difference was seen between obese adolescents with NGT and IGT in liver fat $(P=0.9)$ or intramyocellular fat in the tibialis anterior $(P=0.13)$. In logistic regression analysis, increased soleus intramyocellular fat and intraperitoneal fat were significant predictors of IGT.

Conclusions: IGT in obese adolescents is associated with increased intramyocellular and intraperitoneal fat rather than liver fat.
\end{abstract}

European Journal of Endocrinology $163413-419$

\section{Introduction}

Obesity presenting during childhood is a powerful predictor of adult cardiovascular morbidity independent of adult weight. This is accounted for particularly by the clustering of metabolic syndrome components in obese individuals during childhood (1). Accordingly, obese children might be prone to develop abnormalities of glucose metabolism at a young age. In recent years, it has indeed become increasingly evident that impaired glucose tolerance (IGT), associated with insulin resistance (IR), is a common finding among obese children (2).

Although body mass index (BMI) and total body fat correlate with the risk of IGT and type 2 diabetes during childhood, distribution of body fat may be an even more significant risk factor. Central adiposity is independently associated with IR and dyslipidemia in obese children, but there is controversy on whether intra-abdominal fat or abdominal subcutaneous fat is more specifically associated with impaired insulin sensitivity at a young age $(3,4)$.

Deposition of fat into tissues other than adipose tissue may also be important in glucose metabolism. The accumulation of lipids into muscle cells has been subject to growing interest owing to the critical role of skeletal muscle in insulin-dependent glucose metabolism. The discovery of intramyocellular lipid (IMCL) and extramyocellular lipid (EMCL) measurement by proton magnetic resonance spectroscopy (MRS) has enabled non-invasive differentiation and quantitation of lipid deposits in skeletal muscle (5). The correlation of IMCL with IR was originally described in adults (6), and has subsequently been reported in obese adolescents (7).

Lipid accumulation in the liver also seems to be associated with alterations in glucose metabolism. Non-alcoholic fatty liver disease (NAFLD) is a condition characterized by chronically elevated serum 
aminotransferase levels and hepatic fatty infiltration. NAFLD has been associated with hyperinsulinemia even in the absence of obesity or diabetes (8). Liver fat accumulation can be quantitated by proton spectroscopy, and studies of normal-weight and obese subjects applying this methodology have reported an association of liver fat and IR independently of abdominal and overall obesity (9). Quantitative data on liver fat deposition in children are scarce. However, a recent study reported a high incidence of NAFLD among obese adolescents, in association with increased hepatic and abdominal fat (10).

Severe obesity present at an early age is associated with numerous metabolic disturbances; however, IGT perhaps has a more significant prognostic value. We have previously described an unexpectedly high prevalence of IGT $(40 \%)$ among the severely obese children that have been referred to our clinic (11). The main aim of the present study was to characterize the association between glucose tolerance and intramyocellular, intra-abdominal and liver fat in severely obese adolescents.

\section{Subjects and methods}

\section{Study population}

We invited to this study 26 adolescents, aged 11.5-15.9 years, who had been referred to the Hospital for Children and Adolescents, University of Helsinki, due to severe obesity and had been recruited to a large study of the genetic background and phenotype of childhood obesity (12). Criteria for severe obesity were relative weight-for-height exceeding median for age and sex by at least $60 \%$ (Finnish growth charts) and/or BMI (weight $\left.(\mathrm{kg}) /(\text { height }(\mathrm{m}))^{2}\right) \geq 98$ percentiles by ageadjusted reference tables. Twenty-one of the subjects completed the study protocol, and were thus included in this report. Furthermore, we invited all their normalweight siblings in the age range of 11-17 years to the study. Eight siblings from eight families consented to the study protocol. A written informed consent was obtained from all subjects and from parents of subjects younger than 15 years of age. The study protocol was approved by the ethics committee of the Hospital for Children and Adolescents, University of Helsinki.

Demographic characteristics of the subjects are described in Table 1. Age and sex distribution was similar between non-obese sibling controls and obese subjects.

\section{Biochemical analyses}

A 2-h oral glucose tolerance test was performed at $0800 \mathrm{~h}$ after an overnight fast according to WHO. The subjects ingested 75-g glucose solution, and plasma glucose and serum insulin concentrations were
Table 1 Demographic and auxologic characteristics of non-obese sibling controls and obese children with normal or impaired glucose. Data are mean \pm S.E.M.

\begin{tabular}{lccc}
\hline & $\begin{array}{c}\text { Non-obese } \\
\text { siblings }\end{array}$ & $\begin{array}{c}\text { Obese children } \\
\text { with normal } \\
\text { glucose } \\
\text { tolerance }\end{array}$ & $\begin{array}{c}\text { Obese children } \\
\text { with impaired } \\
\text { glucose } \\
\text { tolerance }\end{array}$ \\
\hline$n$ & 8 & 13 & 8 \\
Sex (M/F) & $5 / 3$ & $7 / 6$ & $5 / 3$ \\
Age (years) & $14.4 \pm 0.7$ & $13.7 \pm 0.4$ & $13.2 \pm 0.5$ \\
Weight (kg) & $62.6 \pm 3.9$ & $95.4 \pm 4.1$ & $96.8 \pm 4.3$ \\
W for H (\%) & $124 \pm 5$ & $190 \pm 7$ & $192 \pm 6$ \\
Height (cm) & $166.8 \pm 5.7$ & $163.4 \pm 2.7$ & $163.8 \pm 3.1$ \\
Height SDS & $+0.7 \pm 0.4$ & $+0.8 \pm 0.3$ & $+1.4 \pm 0.4$ \\
BMl (kg/m $\left.{ }^{2}\right)$ & $22.5 \pm 0.5$ & $35.6 \pm 1.2$ & $36.0 \pm 1.0$ \\
\hline
\end{tabular}

W, weight; $\mathrm{H}$, height

measured from samples obtained at baseline $\left(0^{\prime}\right), 30^{\prime}$, $60^{\prime}$ and $120^{\prime}$. IGT was defined as plasma glucose concentration $\geq 7.8 \mathrm{mmol} / \mathrm{l}$ and $\leq 11.1 \mathrm{mmol} / \mathrm{l}$ at $120^{\prime}$. Diabetes was defined as plasma glucose concentration $\geq 7.0 \mathrm{mmol} / \mathrm{l}$ at $0^{\prime}$ and $/$ or $\geq 11.1 \mathrm{mmol} / \mathrm{l}$ at $120^{\prime}$. Eight obese subjects fulfilled the criteria for IGT.

Samples were also collected at baseline for assessment of fasting plasma or serum total cholesterol, highdensity lipoprotein (HDL) cholesterol, triglycerides, alanine aminotransferase (ALT), urate, free thyroxine, TSH, leptin, cortisol, ACTH and insulin-like growth factor 1 .

\section{Magnetic resonance experiments}

The MR experiments were carried out on a separate visit at the Medical Imaging Centre. The MR imaging (MRI) and MRS studies were performed on a $1.5 \mathrm{~T}$ clinical imager (Siemens, Erlangen, Germany), and consisted of assessments of i) subcutaneous, intra-abdominal and retroperitoneal fat volumes, ii) liver fat content and iii) skeletal muscle lipid stores. The subjects were instructed to follow their typical dietary patterns for 3 days preceding the MR experiment, to avoid any exercise on the day of the investigation, and to not eat anything for the last $2 \mathrm{~h}$ before the investigation.

\section{Abdominal fat MRI}

The subjects lay in a supine position, and a body coil was used to collect sagittal scout images. A stack of 24 T1-weighed (repitition time/echo time (TR/TE) $=$ 91/5.24 ms) trans-axial gradient echo images of $10-\mathrm{mm}$ thickness was centred at the intervertebral disk between the 4 th and 5 th lumbar vertebrae to image intra-abdominal and subcutaneous fat volumes. Data were collected in sets during three breath holds with a total duration of $37 \mathrm{~s}$. Frequency selective fat excitation was used to maximize contrast between the adipose and 
non-adipose tissues. For image segmentation, we used an in-house-built software (13) modified for abdominal volumetry. Images were filtered with a Gaussian function and voxels converted to near isotropic. Sagittal and coronal images were constructed from the near isotropic image voxels. Subcutaneous, intra-abdominal and retroperitoneal fat volumes were manually determined from the three-dimensional image reconstructions in a blinded fashion $(\mathrm{A} \mathrm{H})$.

\section{Hepatic MRS}

Subjects were positioned in a prone position to minimize any respiratory motion of the liver. Coronal, sagittal and transaxial localization images were used to position a $20 \times 20 \times 20 \mathrm{~mm}^{3}$ voxel in the center of the right lobe of the liver avoiding tubular structures, and as far as possible from the surrounding tissues. A PRESS single voxel localization technique with TR/TE of $3000 / 30 \mathrm{~ms}$, spectral width of $1000 \mathrm{~Hz}$ and 128 acquisition was used to obtain liver spectra. The postprocessing and analysis was performed using Siemens syngo standard spectroscopy task card (Siemens). Postprocessing included spectral apodization by Hanning function with a $300 \mathrm{~ms}$ full-width at half-maximum, zerofilling from 1024 to 2048 data points and zero order phase correction. Areas of water (4.7 ppm) and fat $(1.2 \mathrm{ppm})$ peaks were determined using Gaussian lineshape fitting. In the liver spectra line, widths were typically $15-20 \mathrm{~Hz}$ for water resonance, and the $\mathrm{CH}_{2}$ and $\mathrm{CH}_{3}$ peaks were clearly distinguishable. Liver fat \% was calculated by $100 \%$ $\times S_{\text {fat }} /\left(S_{\text {fat }}+S_{\text {water }}\right)$. We also collected in-phase and out-of-phase MRI data in the liver from these individuals and validated the spectroscopic liver fat against in-phase and out-of-phase MRI-data. We found a very strong correlation between results acquired by these two methods $\left(R^{2}=0.925\right)$, showing that potential bleeding of fat signal from outside the liver was not a concern.

\section{Muscle MRS}

In the supine position, the right calf was positioned parallel to main magnetic field in a standard knee coil. Measurements were performed using the PRESS chemical shift imaging (CSI) technique. Field-of-view of $80 \times 80 \mathrm{~mm}$ was covered with $12 \times 12$ phase steps. A $40 \times 40 \times 40 \mathrm{~mm}^{3}$ volume of interest (VOI) was positioned to cover a sample in the middle of the soleus muscle. Water suppressed and non-water suppressed spectra were collected using $500 \mathrm{~Hz}$ spectral width and 2 and 1 averages respectively. The slice selective pulses of PRESS CSI sequence were applied at transmitter frequency of 0 and 2.3 ppm for water suppressed and unsuppressed experiments respectively. This procedure was repeated to measure the tibialis anterior muscle.
For the CSI data, $k$-space was filtered with a $50 \%$ Hamming filter and zero filled up to $16 \times 16$ points leading to a nominal voxel size of $5 \times 5 \times 10 \mathrm{~mm}^{3}$ and Fourier transformed in the spatial dimensions. The time domain data were apodized using a Hamming function with $300 \mathrm{~ms}$ full-width at half-maximum, zero filled once from 512 to 1024 data points, Fourier transformed, and corrected for first and zero order phases. The spectra were analysed using Siemens syngo standard spectroscopy task card (Siemens) in a blinded fashion $(\mathrm{S} \mathrm{H})$. The fitting algorithm works in the frequency domain. The intensity of $\mathrm{H}_{2} \mathrm{O}(4.7 \mathrm{ppm})$ was determined from unsuppressed spectra using Lorenzian lineshape fitting. Water suppressed spectra were used to determine the intensities of EMCL at $1.50 \mathrm{ppm}$, IMCL $1.27 \mathrm{ppm}$ and $\mathrm{Cr}$ signals using Gaussian lineshape fitting. IMCL and EMCL lipid contents were calculated by $100 \% \times S_{\text {fat }} / S_{\text {water }}$. For both soleus and tibialis anterior muscle, 1-8 CSI voxels per muscle were selected for the analysis. Only spectra with a clear separation of the IMCL and EMCL peak tops by a trough were included in signal averaging. Representative spectra are shown in Fig. 1.

\section{Calculations}

IR index based on homeostasis model assessment (HOMA-IR) method was calculated from fasting blood glucose and insulin levels (14). A whole-body insulin sensitivity index (WBISI) was calculated based on fasting and post-glucose load glucose and insulin concentrations using the equation of Matsuda \& DeFronzo (15). Insulogenic index was calculated as AUC (incremental insulin concentration 0-30 min) divided by AUC (incremental glucose concentration 0-30 min).

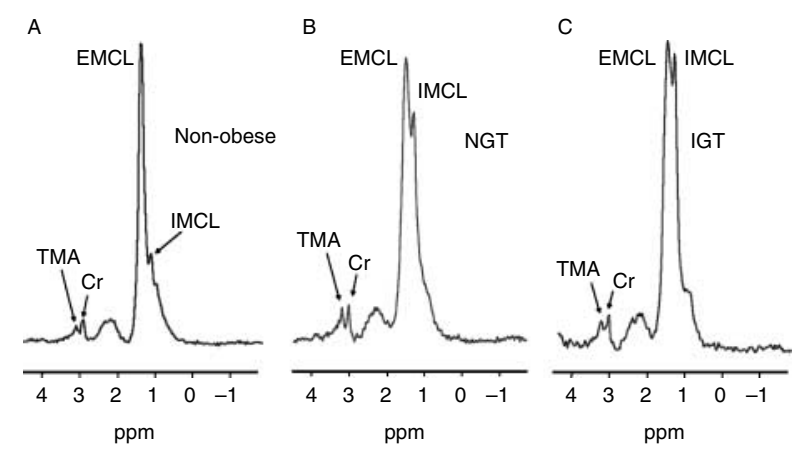

Figure 1 Representative water-suppressed soleus MR-spectra from three individuals; (A) non-obese sibling control; (B) obese subject with normal glucose tolerance (NGT); and (C) obese subject with impaired glucose tolerance (IGT). Intramyocellular fat (IMCL) peak (1.3 ppm) shows as a minute resonance to the right of the dominant extramyocellular fat (EMCL) peak (1.5 ppm) in controls $(A)$, and is present at higher quantities in obese NGT and IGT subjects (B and $\mathrm{C}$ ). TMA, trimethylammonium; $\mathrm{Cr}$, creatine; ppm, parts per million (chemical shift). 


\section{Statistical analyses}

Differences between obese subjects versus non-obese siblings were studied using two-tailed $t$-tests for independent samples. Differences between obese subjects with normal glucose tolerance (NGT) versus IGT in intramyocellular and liver fat and biochemical parameters were studied using analysis of covariance with age, sex and BMI as a covariates (16). Differences in abdominal fat deposits were studied using analysis of covariance with age, sex, weight and height as covariates. The rationale for including weight and height as separate covariates instead of BMI is that subjects with smaller body frames may have a lower amount of intra-abdominal fat than subjects with larger body frames (17). Distributions of the data were examined for normality by the Kolmogorov-Smirnov goodness of fit test. Data that were not normally distributed were logarithmically transformed before statistical analysis.

Data from previous studies showed that a sample size of 12 subjects per group (non-obese siblings/ obese siblings with NGT/obese siblings with IGT) would allow us to detect a $40 \%$ difference in IMCL at a significance level of $95 \%$ and with $80 \%$ power (18).

\section{Results}

\section{Comparison between obese and non-obese siblings}

To determine the effect of obesity on metabolic parameters and distribution of fat deposits, we compared obese adolescents with their non-obese siblings. We found that obese adolescents had significantly impaired fasting and post-load insulin sensitivity as measured by fasting insulin $(P=0.03)$, HOMA-IR $(P=0.03)$ and by WBISI $(P=0.04)$ respectively, and higher levels of leptin $(P=0.003$; Table 2$)$.
Serum lipid concentrations did not differ significantly between obese and non-obese subjects, except for lower HDL-cholesterol levels in obese subjects $(P=0.02)$. Furthermore, obese siblings had significantly increased soleus IMCL $\left(\mathrm{IMCL}_{S} ; P=0.02\right)$, tibialis anterior IMCL $\left(\mathrm{IMCL}_{\mathrm{TA}} ; P=0.01\right)$ and liver fat $(P=0.003)$, as well as increased abdominal subcutaneous $(P<0.001)$, intraperitoneal $(P<0.001)$ and retroperitoneal $(P=0.001)$ fat deposits (Fig. 2).

\section{Comparison between obese adolescents with NGT and IGT}

Biochemical parameters and fat deposition were compared between obese subjects with NGT $(n=13)$ and IGT $(n=8)$. No significant differences were found in biochemical parameters, except for fasting serum leptin concentrations, which were higher in subjects with IGT $(P=0.03$; Table 2). However, patterns of fat depositions were different between the subjects with NGT versus IGT. As shown in Fig. 2, subjects with IGT had significantly increased IMCL $_{S}(P=0.03)$ and intraperitoneal fat $(P=0.04)$. In contrast, no difference was found in liver fat $(P=0.9)$, $\operatorname{IMCL}_{\mathrm{TA}}(P=0.13)$ or abdominal subcutaneous fat $(P=0.13)$ between these groups (Fig. 2).

\section{Correlation and regression analyses}

When both obese and non-obese siblings were included in correlation analysis, liver fat was significantly associated with ALT $(r=0.73, P<0.001)$, BMI $(r=0.52, P=0.006)$, WBISI $(r=0.50, P=0.01)$, fasting insulin $(r=0.47, P=0.01)$ and HOMA $(r=0.44$, $P=0.03)$. However, among obese subjects, liver fat was only associated with ALT $(r=0.73, P<0.001)$, suggesting that these correlations were indicative for association of liver fat with obesity per se rather than

Table 2 Biochemical measurements in non-obese sibling controls and in obese children with normal or impaired glucose tolerance. Data are mean \pm S.E.M.

\begin{tabular}{lcccc}
\hline & Non-obese siblings & Obese children & $\begin{array}{c}\text { Obese children } \\
\text { with normal glucose } \\
\text { tolerance }\end{array}$ & $\begin{array}{c}\text { Obese children with } \\
\text { impaired glucose } \\
\text { tolerance }\end{array}$ \\
\hline$n$ & 8 & 21 & 13 & 8 \\
FP-gluc (mmol/l) & $4.7 \pm 0.1$ & $4.8 \pm 0.1$ & $4.9 \pm 0.1$ & $4.7 \pm 0.2$ \\
FP-ins (mU/l) & $12.3 \pm 2.1$ & $19.4 \pm 1.5^{\star}$ & $20.8 \pm 2.2$ & $17.2 \pm 1.5$ \\
HOMA-IR & $2.6 \pm 0.5$ & $4.1 \pm 0.3^{\star}$ & $4.5 \pm 0.4$ & $3.6 \pm 0.3$ \\
Whole-body insulin sensitivity index & $20.2 \pm 6.1$ & $11.4 \pm 1.1^{*}$ & $12.1 \pm 1.7$ & $10.2 \pm 1.1$ \\
Insulogenic index & $30.1 \pm 4.7$ & $45.0 \pm 6.3$ & $53.0 \pm 9.5$ & $39.9 \pm 3.7$ \\
S-Leptin $(\mu \mathrm{g} / \mathrm{l})$ & $13.4 \pm 4.1$ & $33.3 \pm 2.7^{\dagger}$ & $29.4 \pm 3.7$ & $39.3 \pm 1.1^{\ddagger}$ \\
S-Total cholesterol (mmol/l) & $3.9 \pm 0.3$ & $4.3 \pm 0.2$ & $4.1 \pm 0.2$ & $4.6 \pm 0.6$ \\
S-LDL-chol (mmol/l) & $1.9 \pm 0.5$ & $2.6 \pm 0.2$ & $2.3 \pm 0.2$ & $3.0 \pm 0.5$ \\
S-HDL-chol (mmol/l) & $1.5 \pm 0.1$ & $1.2 \pm 0.0^{\star}$ & $1.2 \pm 0.0$ & $1.1 \pm 0.1$ \\
S-Trigly (mmol/l) & $1.1 \pm 0.2$ & $1.2 \pm 0.2$ & $1.3 \pm 0.3$ & $1.1 \pm 0.2$ \\
S-ALAT (U/l) & $13.1 \pm 1.2$ & $26.0 \pm 3.8$ & $26.9 \pm 5.5$ & $24.6 \pm 5.0$ \\
\hline
\end{tabular}

${ }^{\star} P<0.05,{ }^{\dagger} P<0.01$ versus non-obese siblings. ${ }^{\ddagger} P<0.05$ versus obese children with normal glucose tolerance. 

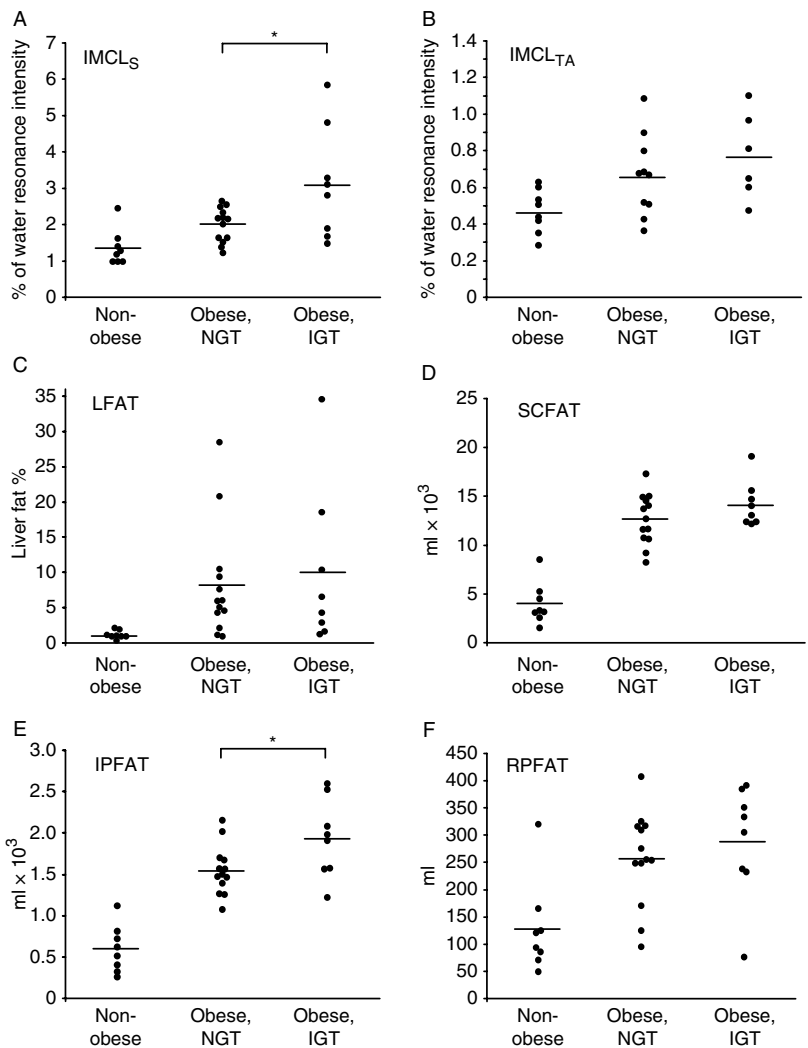

Figure 2 Fat deposits in non-obese adolescents and in obese adolescents with normal (NGT) or impaired glucose tolerance (IGT). (A) Intramyocellular lipid in $\mathrm{m}$. soleus (IMCLs).

(B) Intramyocellular lipid in $\mathrm{m}$. tibialis anterior (IMCL $\mathrm{TA}_{\mathrm{TA}}$ ). (C) Liver fat (LFAT). (D) Abdominal subcutaneous fat (SC fat). (E) Intraperitoneal fat (IPFAT). (F) Retroperitoneal fat (RPFAT). Horizontal lines denote mean values. Significant differences between obese adolescents with NGT or IGT: ${ }^{\star} P<0.05$. Difference between non-obese siblings and obese subjects with NGT or IGT were significant for all parameters $(A-F)$.

with insulin sensitivity. However, there was a significant correlation between liver fat and intraperitoneal fat in obese subjects $(r=0.58, P=0.006)$. Similarly, when all subjects were included, IMCL $_{S}$ showed significant association with leptin $(r=0.45, P=0.04)$ and lowdensity lipoprotein cholesterol $(r=0.43, P=0.03)$ and a weak association with WBISI $(r=-0.33, P=0.099)$, yet among obese subjects no significant correlations were found. In a stepwise logistic regression analysis using likelihood ratio statistics, only increased $\mathrm{IMCL}_{\mathrm{S}}$ and increased intraperitoneal fat came out as significant predictors of IGT.

\section{Discussion}

The key finding of our study is that while obese adolescents clearly have increased IMCL and intrahepatic and intraperitoneal fat deposits compared with their non-obese siblings, only IMCL $_{S}$ and intraperitoneal fat are significantly associated with IGT among the obese subjects. To our knowledge, this study is the first that explores the relationship of all these pathogenic fat deposits with glucose tolerance in obese adolescents. Furthermore, we used an advanced method for the precise quantification of abdominal fat. Abdominal fat deposits have often been measured from single slice data, which is dependent on the correct craniocaudal positioning of the slice and therefore subject to interassay variation. For the present study, we used a three-dimensional method for the assessment of intraperitoneal and retroperitoneal fat volumes.

An association of IMCL with glucose metabolism was originally reported by Krssak et al. (6). In non-obese adults, a significant inverse correlation between IMCL and insulin sensitivity was found, independent of BMI and serum lipid and insulin levels. In a study of healthy men without a family history of type 2 diabetes (T2DM), those subjects whose IMCL was above median had lower whole-body insulin-stimulated glucose uptake, and furthermore, these subjects showed higher serum free fatty acids (FFA) during hyperinsulinemia and defective insulin signalling (19). Interestingly, a dynamic MRS study showed that acute elevation of serum FFA during hyperinsulinemia results in an increase in IMCL (20). In the first study on young subjects (7), IMCL IM $_{S}$ significantly higher in obese than in lean adolescents, and an inverse correlation was found between IMCL and insulin sensitivity. We did not perform the euglycaemic hyperinsulinaemic clamp to assess insulin sensitivity, but chose to use WBISI, an insulin sensitivity index calculated from an oral glucose tolerance test. A strong correlation has earlier been demonstrated between the $M$-values obtained from the clamp and the WBISI in children and adolescents (21). Owing to their invasive nature, the use of the insulin clamp procedures in children and adolescents has been criticized from an ethical point of view (22). Nevertheless, these methodological differences may explain why we found only a weak association between IMCL and insulin sensitivity in our subjects, and might be considered a weakness in our study.

Weiss et al. (23) studied obese children and adolescents with NGT or IGT, and showed an increase in the IMCL of the soleus muscle in the latter. Our results support this finding. The strength in our study is that we analysed IMCL in two types of muscle, soleus and tibialis anterior, and that we also included a control group of non-obese siblings, providing us with more insight into features of IMCL accumulation in early onset obesity and prediabetes. We found that both the soleus $(P=0.009)$ and tibialis anterior $(P=0.01)$ IMCL were significantly increased in the obese IGT subjects compared with their non-obese siblings (Fig. 2). In the obese NGT group, the soleus and tibialis anterior behaved differently; the distribution of the $\mathrm{IMCL}_{\mathrm{S}}$ overlapped markedly with that of the controls, whereas the tibialis anterior had a similar increase in IMCL as the 
obese IGT group. These findings may be explained by the different metabolic characteristics of these muscles $(24,25)$. Thus, in the obese NGT group, there is oversupply of fat in both the soleus and tibialis anterior without any restriction in glucose utilization. In the soleus, where the energy production relies more on fat oxidation, little accumulation of IMCL occurs. On the other hand, in the glycolytic tibialis anterior muscle, the supply of fat exceeds the capacity of lipid oxidation resulting in an accumulation of IMCL.

It had been proposed earlier that increased IMCL in obese adolescents may result from an increased flux of FFAs into the muscle from an enlarged intra-abdominal fat deposit (7). Our findings of increased $\mathrm{IMCL}_{\mathrm{S}}$ and intraperitoneal fat in obese subjects with IGT correlate with this hypothesis. In the earlier study by Weiss et al. (23), intra-abdominal fat was not significantly increased in obese adolescents with IGT, although an increased intra-abdominal-to-subcutaneous fat ratio was demonstrated. The difference between our study and theirs is that we used a three-dimensional method, which allowed us to more precisely assess fat volumes. Our method also enabled us to discern between intraand retroperitoneal fat compartments, yet the importance of this feature may be limited since we found that using our three-dimensional method, total intra-abdominal (intraperitoneal and retroperitoneal) fat was also significantly increased in obese subjects with IGT compared with those with NGT (data not shown).

Studies performed in adult non-obese and obese subjects have suggested a role for liver fat in metabolic syndrome (16) and found that adults with type 2 diabetes have increased liver fat content compared with age- and sex-matched controls (26). In the present study, liver fat was not significantly increased in obese subjects with IGT compared with those with NGT. This finding was not due to insufficient statistical power, since the distributions of liver fat content were almost identical in these groups, and liver fat content exceeding the normal range was found as often among those subjects with NGT as in those with IGT. The obvious difference between the young subjects in our study and obese adults with type 2 diabetes or IGT is that our subjects present with IGT after a remarkably shorter time course of obesity. The results presented here indicate that in severely obese adolescents, IGT is more closely correlated to fat deposited in muscle cells rather than in the liver. Therefore, we suggest that the ectopic deposition of fat in muscle cells could be a marker of a more rapidly progressing deterioration of glucose tolerance and development of diabetes. However, due to the cross-sectional nature of our study, we cannot entirely discount the possibility that an increase in liver fat actually precedes development of IGT in obesity, and therefore, we did not see any difference in liver fat between obese subjects with NGT and IGT. To rule out this explanation, a longitudinal study should be performed in young obese individuals with NGT at baseline and later developing IGT.

In conclusion, our results show that increased intraperitoneal fat and IMCL are associated with IGT in early-onset severe obesity. No significant correlation was found between liver fat and glucose tolerance in obese adolescents. The potential role of fat deposition intraperitoneally and in muscle cells as predictors of type 2 diabetes in this population needs to be investigated in longitudinal studies.

\section{Declaration of interest}

The authors declare that there is no conflict of interest that could be perceived as prejudicing the impartiality of the research reported.

\section{Funding}

This work was supported by the Foundation for Paediatric Research, Finland.

\section{Acknowledgements}

We thank all subjects and their families for taking part in the study.

\section{References}

1 Sinaiko AR, Jacobs DR Jr, Steinberger J, Moran A, Luepker R, Rocchini AP \& Prineas RJ. Insulin resistance syndrome in childhood: associations of the euglycemic insulin clamp and fasting insulin with fatness and other risk factors. Journal of Pediatrics 2001139 700-707.

2 Sinha R, Fisch G, Teague B, Tamborlane WV, Banyas B, Allen K, Savoye M, Rieger V, Taksali S, Barbetta G, Sherwin RS \& Caprio S. Prevalence of impaired glucose tolerance among children and adolescents with marked obesity. New England Journal of Medicine $2002346802-810$.

3 Gower BA, Nagy TR \& Goran MI. Visceral fat, insulin sensitivity, and lipids in prepubertal children. Diabetes 199948 1515-1521.

4 Caprio S, Hyman LD, Limb C, McCarthy S, Lange R, Sherwin RS, Shulman G \& Tamborlane WV. Central adiposity and its metabolic correlates in obese adolescent girls. American Journal of Physiology 1995269 E118-E126.

5 Szczepaniak LS, Babcock EE, Schick F, Dobbins RL, Garg A, Burns DK, McGarry JD \& Stein DT. Measurement of intracellular triglyceride stores by $\mathrm{H}$ spectroscopy: validation in vivo. American Journal of Physiology 1999276 E977-E989.

6 Krssak M, Falk Petersen K, Dresner A, DiPietro L, Vogel SM, Rothman DL, Roden M \& Shulman GI. Intramyocellular lipid concentrations are correlated with insulin sensitivity in humans: a ${ }^{1} \mathrm{H}$ NMR spectroscopy study. Diabetologia 199942 $113-116$.

7 Sinha R, Dufour S, Petersen KF, LeBon V, Enoksson S, Ma YZ, Savoye M, Rothman DL, Shulman GI \& Caprio S. Assessment of skeletal muscle triglyceride content by (1)H nuclear magnetic resonance spectroscopy in lean and obese adolescents: relationships to insulin sensitivity, total body fat, and central adiposity. Diabetes 200251 1022-1027.

8 Marchesini G, Brizi M, Morselli-Labate AM, Bianchi G, Bugianesi E, McCullough AJ, Forlani G \& Melchionda N. Association of nonalcoholic fatty liver disease with insulin resistance. American Journal of Medicine $1999 \mathbf{1 0 7} 450-455$. 
9 Seppala-Lindroos A, Vehkavaara S, Häkkinen AM, Goto T, Westerbacka J, Sovijärvi A, Halavaara J \& Yki-Järvinen H. Fat accumulation in the liver is associated with defects in insulin suppression of glucose production and serum free fatty acids independent of obesity in normal men. Journal of Clinical Endocrinology and Metabolism 200287 3023-3028.

10 Perseghin G, Bonfanti R, Magni S, Lattuada G, De Cobelli F, Canu T, Esposito A, Scifo P, Ntali G, Costantino F, Bosio L, Ragogna F, Del Maschio A, Chiumello G \& Luzi L. Insulin resistance and whole body energy homeostasis in obese adolescents with fatty liver disease. American Journal of Physiology. Endocrinology and Metabolism 2006291 E697-E703.

11 Saukkonen T, Etelämäki-Huhtala P \& Lipsanen-Nyman M. Lower birth weight and reduced insulin sensitivity in markedly obese children with impaired vs. normal glucose tolerance. Hormone Research 200462 (Suppl 2) P1-P230 (Abstract).

12 Valli-Jaakola K, Lipsanen-Nyman M, Oksanen L, Hollenberg AN, Kontula K, Bjørbaek C \& Schalin-Jäntti C. Identification and characterization of melanocortin-4 receptor gene mutations in morbidly obese finnish children and adults. Journal of Clinical Endocrinology and Metabolism 200489 940-945.

13 Van Leemput K, Maes F, Vandermeulen D \& Suetens P. Automated model-based tissue classification of MR images of the brain. IEEE Transactions on Medical Imaging 199918 897-908.

14 Matthews DR, Hosker JP, Rudenski AS, Naylor BA, Treacher DF \& Turner RC. Homeostasis model assessment: insulin resistance and beta-cell function from fasting plasma glucose and insulin concentrations in man. Diabetologia $1985 \mathbf{2 8} 412-419$.

15 Matsuda M \& DeFronzo RA. Insulin sensitivity indices obtained from oral glucose tolerance testing: comparison with the euglycemic insulin clamp. Diabetes Care 199922 1462-1470.

16 Kotronen A, Westerbacka J, Bergholm R, Pietiläinen KH \& Yki-Järvinen $\mathrm{H}$. Liver fat in the metabolic syndrome. Journal of Clinical Endocrinology and Metabolism 200792 3490-3497.

17 Taksali SE, Caprio S, Dziura J, Dufour S, Calí AMG, Goodman TR, Papademetris X, Burgert TS, Pierpont BM, Savoye M, Shaw M, Seyal AA \& Weiss R. High visceral and low abdominal subcutaneous fat stores in the obese adolescent: a determinant of an adverse metabolic phenotype. Diabetes $2008 \mathbf{5 7}$ 367-371.

18 Perseghin G, Scifo P, De Cobelli F, Pagliato E, Battezzati A, Arcelloni C, Vanzulli A, Testolin G, Pozza G, Del Maschio A \& Luzi L.
Intramyocellular triglyceride content is a determinant of in vivo insulin resistance in humans: a ${ }^{1} \mathrm{H}^{-13} \mathrm{C}$ nuclear magnetic resonance spectroscopy assessment in offspring of type 2 diabetic parents. Diabetes $1999 \mathbf{4 8} 1600-1606$.

19 Virkamaki A, Korsheninnikova E, Seppälä-Lindroos A, Vehkavaara S, Goto T, Halavaara J, Häkkinen AM \& Yki-Järvinen H. Intramyocellular lipid is associated with resistance to in vivo insulin actions on glucose uptake, antilipolysis, and early insulin signaling pathways in human skeletal muscle. Diabetes $2001502337-2343$.

20 Brechtel K, Dahl DB, Machann J, Bachmann OP, Wenzel I, Maier T, Claussen CD, Häring HU, Jacob S \& Schick F. Fast elevation of the intramyocellular lipid content in the presence of circulating free fatty acids and hyperinsulinemia: a dynamic ${ }^{1} \mathrm{H}-\mathrm{MRS}$ study. Magnetic Resonance Imaging 200145 179-183.

21 Yeckel CW, Weiss R, Dziura J, Taksali SE, Dufour S, Burgert TS, Tamborlane WV \& Caprio S. Validation of insulin sensitivity indices from oral glucose tolerance test parameters in obese children and adolescents. Journal of Clinical Endocrinology and Metabolism 200489 1096-1101.

22 Mann $H$. Ethics of research involving vulnerable populations. Lancet 2003291857.

23 Weiss R, Dufour S, Taksali SE, Tamborlane WV, Petersen KF, Bonadonna RC, Boselli L, Barbetta G, Allen K, Rife F, Savoye M, Dziura J, Sherwin R, Shulman GI \& Caprio S. Prediabetes in obese youth: a syndrome of impaired glucose tolerance, severe insulin resistance, and altered myocellular and abdominal fat partitioning. Lancet 2003362 951-957.

24 Schrauwen-Hinderling VB, Hesselink MK, Schrauwen P \& Kooi ME. Intramyocellular lipid content in human skeletal muscle. Obesity 200614 357-367.

25 Polgar J, Johnson MA, Weightman D \& Appleton D. Data on fibre size in thirty-six human muscles. An autopsy study. Journal of Neurological Sciences 197319 307-318.

26 Kotronen A, Juurinen L, Hakkarainen A, Westerbacka J, Cornér A, Bergholm R, Yki-Järvinen $\mathrm{H}$ \& Hakkarainen A. Liver fat is increased in type 2 diabetic patients and underestimated by serum alanine aminotransferase compared with equally obese nondiabetic subjects. Diabetes Care 200831 165-169.

Received 23 June 2010

Accepted 28 June 2010 